\title{
Relationship of DUX4 and target gene expression in FSHD myocytes
}

Jonathan Chau, ${ }^{1}$ Xiangduo Kong, ${ }^{1}$ Nam Nguyen, ${ }^{1}$ Katherine Williams, ${ }^{2}$ Rabi Tawil, ${ }^{3}$

Tohru Kiyono, ${ }^{4}$ Ali Mortazavi, ${ }^{2 *}$ and Kyoko Yokomori ${ }^{1 *}$

${ }^{1}$ Dept. Biol. Chem., School of Med., Univ. California, Irvine, CA

${ }^{2}$ Dept. Dev. Cell Biol., School of Biol. Sci., Univ. California, Irvine, CA

${ }^{3}$ Neuromuscular Disease Unit, Department of Neurology, University of Rochester

Medical Center, Rochester, New York, USA

${ }^{4}$ Exploratory Oncology Research \& Clinical Trial Center, National Cancer Center, Kashiwa City, Chiba, Japan

${ }^{*}$ co-corresponding authors: Ali Mortazavi, 2218 Biological Sciences III, Department of Developmental and Cell Biology, School of Biological Sciences, University of California, Irvine, CA92697-2300, U.S.A.; TEL: 949-824-6762; EMAIL: ali.mortazavi@uci.edu ; Kyoko Yokomori, 240D, Medical Sciences I, Department of Biological Chemistry, School of Medicine, University of California, Irvine, CA92617-1700, U.S.A.; TEL: 949-824-8215; EMAIL: kyokomor@uci.edu 


\section{Abstract}

Facioscapulohumeral dystrophy (FSHD) is linked to misexpression of the transcription factor, DUX4. Although DUX4 target gene expression is often readily detectable, analysis of DUX4 expression has been limited due to its low expression in patient samples. Recently, single cell/nucleus RNA-sequencing was used to detect the native expression of $D U X 4$ for the first time, but important spatial relationships with its target gene expression was missing. Furthermore, dynamics of $D U X 4$ expression during myoblast differentiation has not been fully explored. In order to study the spatiotemporal relationship of DUX4 and key target genes, we performed RNA FISH on immortalized FSHD2 patient skeletal muscle cells. Using two probe sets, DUX4 transcripts were detected in 1-4\% of myotubes after 3-day differentiation in vitro. We found that $D U X 4$ transcripts mainly localize as foci in one or two nuclei in a myotube compared to abundant accumulation of the target gene transcripts in the cytoplasm.

Over a 13-day differentiation timecourse, DUX4 expression without target gene expression significantly increased and peaked at day 7 . Target gene expression correlates better with $D U X 4$ expression early in differentiation while the expression of target genes without detectable $D U X 4$ transcripts increases later. Consistently, shRNA depletion of DUX4-activated transcription factors, DUXA and LEUTX, specifically repressed a DUX4-target gene, KDM4E, later in differentiation, suggesting that following the initial activation by DUX4, target genes themselves contribute to the maintenance of downstream gene expression. Together, in situ detection of the DUX4 and target gene transcripts provided new insight into dynamics of DUX4 transcriptional network in FSHD patient myocytes. 
bioRxiv preprint doi: https://doi.org/10.1101/2020.05.24.109710; this version posted May 25, 2020. The copyright holder for this preprint (which was not certified by peer review) is the author/funder. All rights reserved. No reuse allowed without permission.

Keywords: FSHD, DUX4, LEUTX, KDM4E, RNAScope, skeletal myotubes 


\section{Significance Statement}

FSHD is the third most common muscular dystrophy and is associated with upregulation of $D U X 4$, a transcription factor, and its target genes. Although target genes are easily detectable in FSHD, low frequency DUX4 upregulation in patient myocytes is difficult to detect, and examining the relationship and dynamics of DUX4 and target gene expression without artificial overexpression of DUX4 has been challenging. Using RNAScope with highly specific probes, we detect the endogenous DUX4 and target gene transcripts in situ in patient skeletal myotubes during differentiation in vitro. Our study reveals a unique $D U X 4$ expression pattern and its relationship to the expression of target genes, and evidence for self-sustainability of the target gene network. The study provides important new insights into the FSHD disease mechanism. 


\section{Introduction}

Facioscapulohumeral dystrophy (FSHD) is an autosomal dominant muscular dystrophy initiating with progressive wasting of facial, shoulder, and upper arm musculature (1). It is one of the most common muscular dystrophies (1 in 8,333$)(2)$. The majority of FSHD cases $(>95 \%)$ are linked to monoallelic deletion of D4Z4 macrosatellite repeat sequences at the subtelomeric region of chromosome $4 \mathrm{q}$ (4qter D4Z4) (termed FSHD1 (MIM 158900)) (1, 3). Only one to ten D4Z4 repeats are found in the contracted allele in FSHD1 while 11 150 copies are present in the intact allele. FSHD2 is the rare form of FSHD ( $<5 \%$ of cases) with no D4Z4 repeat contraction but exhibits the clinical phenotype identical to FSHD1 (4). Recent studies have found that the SMCHD1 gene is mutated in $>80 \%$ of FSHD2 cases (MIM 158901) (5) as well as in severe cases of $\operatorname{FSHD1}(6,7)$.

D4Z4 is a $3.3 \mathrm{~kb}$ repeat containing an open reading frame for the double-homeobox transcription factor $D U X 4$ gene (8-10). DUX4 is essential during early embryogenesis but is subsequently silenced $(11,12)$. Only individuals with a $4 q A$ haplotype with a polyadenylation signal sequence for the DUX4 transcript distal to the last D4Z4 repeat express a full-length DUX4 transcript (DUX4fl) and develop FSHD (13). Expression of $D U X 4 f l$ is closely associated with FSHD, which strongly suggests that DUX4 expression is critical for FSHD pathogenesis $(10,13,14)$. Activation of many, if not all, DUX4 target genes has been observed in patient cells in multiple studies, supporting the significance of DUX4fl in FSHD. However, how dysregulation of any of these target genes directly contributes to the disease process is still under active investigation $(9,15-19)$. 
Curiously, the DUX4fl transcript is expressed at extremely low levels and sometimes is not detectable $(10,17)$, and DUX4 protein is detectable only in $<0.1 \%$ of patient muscle cells $(10,13,14,20)$. Furthermore, DUX4fl expression can occasionally be observed even in unaffected individuals $(10,17)$. Although overexpression of the recombinant DUX4 in in vitro myoblasts and in vivo in model organisms was shown to be toxic $(21,22)$, recent evidence indicates that the phenotype induced by the recombinant overexpression can differ from that of the endogenous DUX4 (23). Thus, there is a critical need to study the effect of the endogenous $D U X 4$ expression. However, assessment of the endogenous DUX4 and target gene expression in FSHD patient myocytes has been limited. Recently, we detected DUX4 and target gene transcripts using single-nucleus RNA-seq (snRNA-seq) (24). Unlike the previous single cell RNA-seq of fusion-blocked myotubes (25), our isolation and analyses of nuclei from naturally fused multi-nucleated myotubes provided the first evidence that DUX4 target gene expression is much more wide-spread than DUX4 transcription itself, which explains easier detection of the target gene transcripts rather than DUX4 itself $(9,15-$ 19). SnRNA-seq was highly instrumental in defining the different states of FSHD patient myocyte nuclei distinct from those of control myocyte nuclei. However, it failed to provide spatial relationship of individual nuclei and associated gene expression. In the current study, we examined the spatiotemporal relationship between the expression of DUX4 and some of its major target genes in control and FSHD2 myocytes during differentiation using RNAScope, an in situ hybridization assay for RNA detection. We designed the probe set that maximizes the potential to detect $D U X 4 f l$ and minimizes the crossreactivity with other isoforms and related genes. Our results provide the first direct 
visualization of $D U X 4$ and target gene activation in patient myocytes during differentiation as well as evidence for DUX4 target transcription factors controlling another DUX4 target gene. These results serve as an important basis for further understanding of the FSHD pathogenesis. 


\section{Results and Discussion}

\section{DUX4 RNA accumulates in the nucleus of the FSHD myotubes}

Multiple DUX4 homologs and isoforms are known to be expressed in human myocytes (10). In particular, DUX4s and DUX4c were shown to be expressed more widely than DUX4fl even in control cells (Snider et al. 2010; Ansseau et al. 2009) (Supplemental Figure S1). Thus, to minimize crossreactivity and maximize the preferential detection of the full length $D U X 4$ (DUX4fl) shown to be most relevant to FSHD, we custom designed an RNAScope probe set with the lowest possible number of ZZ probe pairs ("ZZ" represents a pair of RNAScope target probes) for the fluorescent detection system (Fig. 1A;6ZZ). Interestingly, we observed the major DUX4 transcript signal as foci in the nucleus using this set in primary FSHD myotubes (Fig. 1B) (24).

Unlike our probe set, previous studies using either a conventional FISH probe or another RNAScope probe set (ACDBio cat. no. 498541) detected DUX4 transcript signals in the cytoplasm $(26,27)$. To address this apparent discrepancy, we performed costaining of the two RNAScope probe sets. The previous probe set was designed for the colorimetric DAB staining (26), which does not allow costaining. Thus, we remade the previous probe set compatible with fluorescent labeling and performed costaining with our new $6 Z Z$ probe set. As expected, fluorescent labeling do not give as strong a signal as DAB (Fig. 1B) (26). Since the previous DUX4 probe set contains $15 \mathrm{ZZ}$ pairs (thus designated 15ZZ), the fluorescent signal is stronger than our $6 Z Z$ probe set (Fig. $1 \mathrm{~B}$ and $\mathrm{C})$. Weaker staining by $6 Z Z$ is due to fewer $\mathrm{ZZ}$ pairs in our probe set in order to 
minimize the potential crossreactivity to $D U X 4 s$ and $D U X 4 c$ (Fig. 1A). In the $15 Z Z$ probe set, 3 and $5 Z Z$ pairs reside in two sub-regions of DUX4fI RNA shared by DUX4s and $D U X 4 c$ transcripts, enabling $4 Z Z$ and $6 Z Z$ pairs to cross-hybridize $D U X 4 s$ and DUX4c, respectively (Fig. 1A; 15ZZ). Consistent with this, $15 Z Z$ shows some staining in control cells, in contrast to $6 Z Z$ which has no significant signal (Fig. 1C). It is therefore possible that some of the signals detected by the $15 Z Z$ probe set may come from that of DUX4s and/or DUX4c. Nevertheless, we found close colocalization of major fluorescent signals at nuclear foci by both probe sets, confirming the significant retention of $D U X 4$ transcripts in the nucleus (Fig. 1B). Some nuclear staining was also apparent in Figure $2 \mathrm{C}$ in the previous paper (26) though it was less clear due to the dark nuclear hematoxylin staining. We further confirmed the presence of DUX4 protein in the same myotube that contains the endogenous DUX4 transcript-positive nuclei (Fig. 1D). Consistent with previous observations $(19,24,28)$, expression of DUX4 RNA transcripts in even one nucleus appears to be sufficient for DUX4 protein localization in most of the nuclei in the same myotube. Taken together, these results strongly support the specificity of the $6 \mathrm{ZZ}$ probe set and indicate that the majority of $D U X 4$ transcripts are retained in the nucleus forming foci in FSHD myotubes.

Cytoplasmic signals can also be observed by the $15 Z Z$ probe set and weakly by ours in some FSHD myotubes with higher expression of endogenous DUX4 (Fig. 1B, indicated by arrowheads). Since the cytoplasmic localization of the recombinant $D U X 4$ (rDUX4) transcript was observed previously (26), we also examined the localization of $r D U X 4$ transcript. Indeed, we found that the overexpressed rDUX4 transcript is highly localized in the cytoplasm with much less nuclear localization (Supplemental Fig. S2). It 
is currently unclear whether this different RNA localization pattern is simply due to vast overexpression of the recombinant DUX4 or possibly due to differences in template sequences and/or gene locations (ectopic vs. genomic). It is possible, for example, that untranslated regions, which are missing in the recombinant $D U X 4$ construct, may dictate the nuclear localization of the endogenous DUX4 transcript. Nevertheless, the results revealed the significant difference between the endogenous and overexpressed recombinant $D U X 4$ transcript localization, highlighting the distinct retention in the nucleus of DUX4 RNA transcribed from the endogenous locus.

\section{Quantification of DUX4fI-expressing nuclei in immortalized FHSD myotubes}

To perform systematic analyses of DUX4 and target gene transcripts, we established immortalized control and FSHD2 myoblast lines using $h T E R T$, mutant CDK4 and CCND1 as described previously (29). We chose one particular patient myoblast sample for immortalization because of the significant expression of DUX4 and target genes, which were closely characterized by snRNA-seq (24). After immortalization and surface marker isolation, several single clones were characterized and compared, and one of them that retained high proliferation and differentiation capabilities was chosen and used for the rest of the study. Using the immortalized FSHD myotubes on day 4 of differentiation (Fig. 2A), we observed similar nuclear foci of DUX4 RNA by both $15 Z Z$ and $6 Z Z$ probe sets as in primary cells (Fig. 1), indicating that immortalization did not affect DUX4 RNA expression and localization (Fig. 2B). We further quantified DUX4 RNA signals detected by $6 Z Z$ in control and FSHD myotubes. No signal was detected in control myotubes $(\mathrm{N}=348)$ (Fig. 2C). We observed DUX4 
RNAScope signals in one to two nuclei in $\sim 2.8 \%$ of FSHD2 myotubes on average (N=414) (Fig. 2C). Comparable DUX4 RNAScope signal patterns and frequencies were observed in both primary parental and corresponding immortalized myotubes (Figs. 1 and 2) (24). Similar to the primary cells, we observed that all nuclei in a myotube are positive for DUX4 protein when some of the nuclei express DUX4 RNA (Figs. 1D and 2D).

\section{Time course analyses of DUX4 and target gene transcripts}

To examine the relationship between DUX4 and its target transcripts, we designed probes for LEUTX, KDM4E, ZSCAN4, and SLC34A2 transcripts known to be activated by DUX4 $(19,30,31)$. We found specific expression of LEUTX, KDM4E, and ZSCAN4 using our RNAScope probe sets in FSHD myotubes in a frequency similar to that of DUX4 (Figs. 2C and 3A). We failed to detect any significant target gene signals in undifferentiated myotubes (data not shown). Unlike $D U X 4$, however, these target gene transcripts, if expressed, accumulate abundantly in the cytoplasm (Fig. 3A). The probe set for SLC34A2 detected strong cytoplasmic signals in FSHD myotubes (Fig. 3A). However, some non-specific staining was seen in control cells, and thus the probe set appears to be not completely specific to FSHD-induced SLC34A2 RNA (data not shown). Thus, we eliminated this probe from further analyses. We confirmed the presence of LEUTX protein in all the nuclei in the same myotube with LEUTX RNA signal, confirming the correlation of RNA and protein expression (Fig. 3B, middle). We observed one myotube with weak LEUTX protein staining and no RNA (Fig. 3B, bottom). It is unclear whether this represents a myotube with residual LEUTX protein after mRNA 
transcription was ceased. With these results, we chose LEUTX and KDM4E transcripts for further time course analyses.

While the upregulation of DUX4 target genes in FSHD is relatively well established, how DUX4 activation results in target gene expression during human myoblast differentiation has not been studied at the single cell level in the context of the natively fused myotubes. Previous work to understand this question includes single cell RNA-seq done on fusion-inhibited myocytes (25) and our recent single-nucleus RNAseq (24). However, neither study was able to address dynamics of DUX4 and target gene expression in the intact myotube. Thus, we performed triple staining for endogenous DUX4, LEUTX, and KDM4E expression to investigate their localization in situ during differentiation from day 3 through day 13 (Fig. 4 and Supplemental Figure S3). Because the number of all DUX4 and target gene-expressing myotubes is limited (typically $\sim 2 \%$ for days $3-5$ and $\sim 5 \%$ for later days of the entire myotube population on a coverslip), obtaining statistically significant quantification data is challenging. Nevertheless, with multiple experimental replicates, we were able to observe a reproducible trend for $D U X 4$ and target gene expression kinetics during differentiation (Fig. 4B). Previously, we followed the increase of DUX4 target gene induction up to 5 days of differentiation by RNA-seq (24). In the current study, we observed that the number of myotubes expressing target genes (LEUTX and/or KDM4E with or without $D U X 4)$ has a tendency to increase throughout the duration of a 13-day time course (Fig. 4C, pink and blue). Increasing trajectory of DUX4 and target gene-positive myotubes argue against the previous suggestion that DUX4 expression leads to immediate cell death $(19,32)$. We also found that the number of myotubes with $D U X 4$ only expression 
(without any target gene expression) increases significantly starting at day 3 and peaks at day 7 (Fig. 4B and $\mathrm{C}$ green), indicating that there are two states of DUX4-positive myotubes (with or without downstream gene activation). The results raise the possibility that an additional factor(s) may be involved in efficient downstream target gene activation.

Unexpectedly, the number of myotubes expressing two target genes (LEUTX and/or KDM4E) without DUX4 increased later in differentiation (Fig. 4C blue), suggesting their continued upregulation with no DUX4 transcripts present. Consistent with this, the frequency of LEUTX coexpression with DUX4 decreases significantly later in differentiation (Fig. 4D). These results strongly suggest that for those myotubes in which the target genes are activated (initially by DUX4), their expression may continue even when DUX4 expression is not maintained. Taken together, the results reveal some discordance between DUX4 and target gene expression, suggesting the possible presence of additional regulatory mechanisms for the DUX4 gene network activation in FSHD patient myocytes.

\section{KDM4E expression is regulated by DUXA and LEUTX}

The above results reveal coexpression of DUX4 target genes in the same myotubes with no detectable DUX4 (Fig. 4B and C). In particular, the number of KDM4E expressing myotubes increases even after DUX4 expression peaks at day 7

(Fig. 5A). Coexpression of KDM4E and LEUTX without DUX4 increases later in differentiation (Fig. 4C, blue) and KDM4E transcript expression correlates better with expression of LEUTX than DUX4 (Fig. 5B). Costaining of LEUTX protein with KDM4E 
transcripts on day 6 revealed that all observed KDM4E staining co-localized with LEUTX protein staining in the same myotubes (Supplemental Fig. S4). Motif analyses revealed that the promoter region of the KDM4E gene contains not only the DUX4 binding motif, but also multiple sites of the putative LEUTX binding motif (Supplemental Fig. S5) $(33,34)$. These results raise the possibility that LEUTX may be involved in KDM4E upregulation. We recently found that DUXA, another DUX4 target, plays a significant role in upregulating LEUTX later in differentiation (24). Thus, we depleted LEUTX and DUXA by shRNAs (Fig. 5C). Because of the difficulty detecting DUXA and LEUTX proteins by western blot, depletion efficiency was confirmed by RT-qPCR (Fig. 5D). Although depletion efficiency is at a comparable level on day 4 and day 6 of differentiation, LEUTX and DUXA depletion specifically repressed KDM4E expression on day 6 , but not day 4 . While we do not detect DUXA motifs in the promoter of KDM4E, we cannot preclude DUXA binding given the similarity of its motif to that of DUX4. Taken together, these results indicate that LEUTX (and DUXA either directly or indirectly through LEUTX upregulation) promotes KDM4E expression following the initial activation by DUX4 (Fig. 5E). The results support our hypothesis that once activated, the DUX4 target genes may in part self-sustain their expression (24).

Detection of the endogenous $D U X 4$ expression and its relationship with its target gene expression in patient myocytes has been technically challenging due to the low frequency of DUX4 expressing cells. Here we use a custom-designed RNAScope probe set to maximize the detection of the pathogenic full-length DUX4 transcript and to analyze its localization and relationship with its downstream target genes in 
differentiating FSHD myotubes. The use of RNAScope ((26) and the current study) provides a complementary tool to single-cell/nucleus RNA-sequencing, to understand FSHD pathogenesis with high spatiotemporal resolution. Our results provide snapshots of FSHD-induced gene expression in patient myocytes during differentiation, revealing that (1) the endogenous DUX4 RNA mainly accumulates in the nucleus; (2) DUX4 expression increases over a week of differentiation, suggesting that it is not immediately toxic; (3) DUX4 and target gene expression are not always concordant, identifying different states of DUX4-positive myotubes; and (4) once activated by DUX4, target genes by themselves can contribute to sustaining DUX4-induced gene expression changes, and that LEUTX, a primate-specific transcription factor like DUX4, is involved in this process. Taken together, our findings provide new insight into gene expression changes in patient myocytes, and evidence for an additional layer of regulation to the DUX4-induced transcriptional network. 


\section{Methods}

\section{Cell culture and differentiation}

Primary and immortalized control and FSHD2 skeletal myoblast cells were grown in high glucose DMEM (Gibco) supplemented with 20\% FBS (Omega Scientific, Inc.), 1\% Pen-Strep (Gibco), and 2\% Ultrasor G (Crescent Chemical Co.). Primary control and FSHD2 myoblasts (24) were immortalized using hTERT with p16INK4a-resistant R24C mutant CDK4 (mtCDK4) and Cyclin D1 as previously described (35). After immortalization, CD56-positive cells were selected by magnetic-activated cell sorting conjugated with anti-CD56 antibody (130-050-401, MiltenyiBiotec). Single cell clones were isolated by FACS sorting into 96 well plates. Control and FSHD2 clones were chosen for the experiments based on normal doubling time and high differentiation index. Myoblast differentiation was induced as previously described (29). Briefly, cells were plated at a seeding density of $\sim 2.5 \times 10^{5}$ cells $/ \mathrm{ml}$ in $0.5 \mathrm{ml}$ of growth medium in each well of a 24-well dish. Approximately 12-16 hr later differentiation was induced using high glucose DMEM medium supplemented with $2 \%$ FBS and ITS supplement (insulin $0.1 \%, 0.000067 \%$ sodium selenite, $0.055 \%$ transferrin, 51300044 Invitrogen). Fresh differentiation medium was changed every day.

\section{Antibodies and cDNA clone}

Immunofluorescence was performed using rabbit polyclonal antibodies specific for DUX4 (ab124699, Abcam) and LEUTX (PA5-59595, Thermofisher). The recombinant DUX4 expression plasmid (pCS2-mkgDUX4) was a gift from Dr. Stephen Tapscott 
(Addgene plasmid \# 21156) (36).

\section{RNAScope probe design}

The following RNAScope probes (Advanced Cell Diagnostics, Inc.) were used: LEUTX (Hs-LEUTX-C2, Cat No. 547251-C2), KDM4E (Hs-KDM4E-C3, Cat No. 556121-C3), SLC34A2 (Hs-SLC34A2-C3, Cat No. 407101-C3), ZSCAN4 (Hs-ZSCAN4-C2, Cat No. 421091-C2) and $15 Z Z$ DUX4 (Hs-DUX4-No-XMm-C3, Cat No. 498541-C3). The 6ZZ DUX4fl probe set (HS-DUX4-O6-C1, Cat No. 546151) was specifically designed for DUX4fI (NM_001306068.2), with only 1 or 2 ZZ pairs residing in the regions shared with DUX4s or DUX4c (at least 3ZZs are needed for signal detection) (Fig. 1A).

\section{RNAScope hybridization}

Cells were grown and differentiated on cover slips in 24-well plates. Cells were washed twice with PBS, then fixed with 10\% neutral buffered formalin (NBF) for 30 min at room temperature (RT), and finally dehydrated with $50 \%, 70 \%$, and $100 \%$ ethyl alcohol gradients for 1 min each at room temperature. Cells were then rehydrated with $70 \%$ and $50 \%$ ethyl alcohol gradients for 1 min each and finally treated with PBS for $10 \mathrm{~min}$. Cells were then treated with hydrogen peroxide (Cat No. 322335 ACDBio) and protease III (Cat No. 322337 ACDBio) at RT for 10 min each and washed with PBS. The probe sets for DUX4, LEUTX, KDM4E were then added in a $50: 1: 1$ ratio for $2 \mathrm{~h}$ at $40^{\circ} \mathrm{C}$ within a humidity control chamber. Probe sets are optimized for different fluorescent channels (C1, C2, and C3, respectively). RNAScope multiplex signal amplification (Cat No. 323110 ACDBio) were applied sequentially and incubated in AMP 1, AMP 2, AMP 3 for 
$30,30,15$ min respectively at $40^{\circ} \mathrm{C}$ within the humidity control chamber. Before adding each AMP reagent, cells were washed twice with RNAScope washing buffer (Cat No. 310091 ACDBio). Signal was developed with the Fluorescent Detection Reagents (Cat No. 323110 ACDBio) and TSA Plus Fluorophores diluted 1:1500 in RNAScope TSA Buffer (Cat No. 322810 ACDBio). To develop C1 signal, samples were incubated in HRP-C1, TSA Plus Fluorescein (cat. No. NEL741001KT PerkinElmer), HRP Blocker for $15,30,15 \mathrm{~min}$, respectively, at $40^{\circ} \mathrm{C}$ within the humidity control chamber. Before adding each reagent, cells were washed twice with RNAScope washing buffer. For C2 and C3 costaining, TSA Plus Cy 3 and Cy 5 were used, respectively (cat no. NEL744001KT and NEL745001KT PerkinElmer). Cells were then counterstained with DAPI (Cat No. 320858 ACDBio) for 30s at RT and washed twice with PBS. Samples were then dried and mounted onto microscope slides with ProLong Diamond Antifade Mountant (Cat No. P36961 ThermoFisher).

\section{Immunofluorescent co-staining with RNAScope}

Cells were grown and differentiated on coverslips in 24-well plates. For costaining of

DUX4 antibody and DUX4 RNAScope probes, cells were fixed with 10\% NBF for 30 min at RT and then extracted with $0.5 \%$ Triton X-100 in PBS. Primary and secondary antibodies were diluted in SNBP (1XPBS $/ 0.02 \%$ saponin $/ 0.05 \% \mathrm{NaN}_{3} / 1 \% \mathrm{BSA}$ ), containing $1 \%$ horse serum and $0.05 \%$ gelatin. Samples were incubated in primary antibody for $30 \mathrm{~min}$ at $37^{\circ} \mathrm{C}$ followed by three PBST (PBS with $0.05 \%$ Tween20) washes. Coverslips were incubated in secondary antibody for $30 \mathrm{~min}$ at $37^{\circ} \mathrm{C}$ followed by three PBST washes. Cells were then dehydrated with $50 \%, 70 \%$, and $100 \%$ ethyl 
alcohol gradients for 1 min each at RT and continued to be processed for RNAScope hybridization as described above. Then coverslips were counterstained with DAPI, washed with $\mathrm{dH}_{2} \mathrm{O}$ and mounted with Prolong Diamond Antifade Mountant. For costaining of LEUTX protein and LEUTX RNA, the RNAscope protocol was done before the immunofluorescence. Before DAPI counterstaining in the RNAscope procedure, coverslips were blocked in PBST, containing 2\% BSA and 10\% Milk for 45 min. Primary and Secondary antibodies were diluted in the blocking buffer. Samples were incubated in primary antibody for $2 \mathrm{~h}$ at RT followed by three PBST washes. Coverslips were then incubated in secondary antibody for $1 \mathrm{~h}$ at RT followed by three PBST washes. Then, coverslips were counterstained with DAPI, washed with PBST and mounted with Prolong Diamond Antifade Mountant.

\section{Fluorescent image acquisition, quantification and statistical analysis}

Images were acquired with a Zeiss LSM510 confocal laser microscope. On the $12 \mathrm{~mm}$ diameter coverslip, 5 horizontal sections were observed and myotubes were counted. Three replicates were done of each staining experiment and error bars were calculated using standard deviation from the mean.

\section{ShRNA depletion and RT-qPCR}

Lentiviruses carrying shRNA plasmids (MISSION shRNA, Sigma-Aldrich) for each DUX4 target gene: DUXA (5'-CTAGATTACTTCTCCAGAGAA-3', TRCN0000017664), LEUTX (5'-CCTGGAATCTCTGATGCAAAT-3', TRCN0000336862), and an shRNA control (SHC002) were made in 293T cells using Lipofectamine P3000. The cells were 
transfected with $2 \mu \mathrm{g}$ of shRNA plasmids, $1.5 \mu \mathrm{g}$ of pCMV plasmids, and $0.5 \mu \mathrm{g}$ of pMP2G plasmids (37). The media was changed after 24 hours. The lentiviruses were harvested at 48 hour and 72 hour post-transfection. FSHD2 immortalized myoblasts were infected twice at 32 hour and 8 hour prior to differentiation. The myoblasts were selected with puromycin. The RNA was extracted using RNeasy kit (Qiagen, Cat No. 74134 ) at days 4 and 6 . Around $16 \mathrm{ng}$ of RNA was converted to cDNA using SuperScript IV VILO Master (Thermofisher, Cat No. 11756050) and then used for RTqPCR analysis. PCR primers are listed in Supplemental Table S1.

\section{Statistical Analyses}

Microsoft Excel software was used to perform statistical analyses on data from three independent experiments. The statistical significance is determined by the Student's ttest. $P<0.05$ is considered of statistical significance. The error bars denote standard deviation. Phi $(\varphi)$ coefficient (analytically equivalent to Pearson's correlation for binary data) is calculated following the previous paper (38). $\varphi$ takes on values ranging between +1 and -1 . The following points are the accepted guidelines for interpreting the

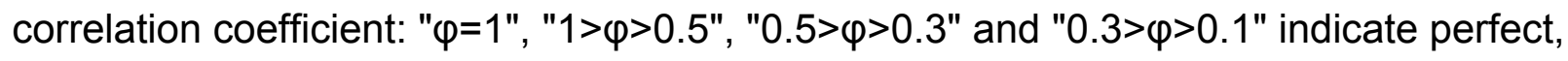

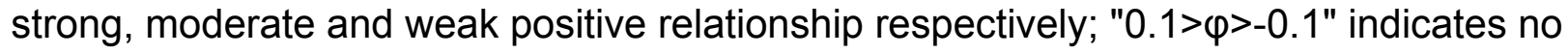

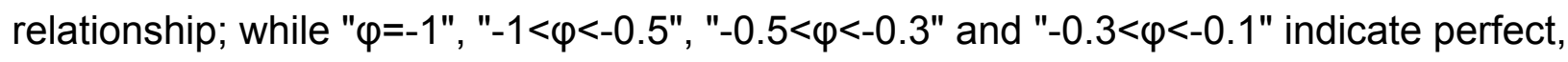
strong, moderate and weak negative relationship respectively. 


\section{Acknowledgement}

The authors wish to acknowledge the support of the Chao Family Comprehensive

Cancer Center Optical Biology Core (LAMMP/OBC) Shared Resource. We would also

like to thank Dr. Naohiro Hashimoto (National Center for Geriatrics and Gerontology, Japan) for technical advice and Kevin Cabrera for helpful comments on the manuscript.

This work was supported in part by National Institutes of Health (P01NS069539 to R.T., and R01AR071287 to K. Y. and A. M.). 


\section{References}

1. van der Maarel SM \& Frants RR (2005) The D4Z4 repeat-mediated pathogenesis of facioscapulohumeral muscular dystrophy. Am. J. Hum. Genet. 76:375-386.

2. Deenen JC, et al. (2014) Population-based incidence and prevalence of facioscapulohumeral dystrophy. Neurol. 83:1056-1059.

3. van der Maarel SM, Tawil R, \& Tapscott SJ (2011) Facioscapulohumeral muscular dystrophy and DUX4: breaking the silence. Trends Mol. Med. 17:252-258.

4. de Greef JC, et al. (2010) Clinical features of facioscapulohumeral muscular dystrophy 2. Neurol. 75:1548-1554.

5. Lemmers RJLF, et al. (2012) Digenic inheritance of an SMCHD1 mutation and an FSHDpermissive D4Z4 allele causes facioscapulohumeral muscular dystrophy type 2. Nat. Genet. 44:1370-1374.

6. Sacconi S, et al. (2013) The FSHD2 Gene SMCHD1 Is a Modifier of Disease Severity in Families Affected by FSHD1. Am. J. Hum. Genet. 93:744-751.

7. Larsen M, et al. (2015) Diagnostic approach for FSHD revisited: SMCHD1 mutations cause FSHD2 and act as modifiers of disease severity in FSHD1. Eur. J. Hum. Genet. 23:808-816.

8. Gabriëls J, et al. (1999) Nucleotide sequence of the partially deleted D4Z4 locus in a patient with FSHD identifies a putative gene within each 3.3 kb element. Gene 236:2532.

9. Geng LN, et al. (2012) DUX4 Activates Germline Genes, Retroelements, and Immune Mediators: Implications for Facioscapulohumeral Dystrophy. Dev. Cell 22:38-51. 
10. Snider L, et al. (2010) Facioscapulohumeral dystrophy: incomplete suppression of a retrotransposed gene. PLoS Genet. 6:e1001181.

11. Whiddon JL, Langford AT, Wong CJ, Zhong JW, \& Tapscott SJ (2017) Conservation and innovation in the DUX4-family gene network. Nat. Genet. 49:935-940.

12. Hendrickson PG, et al. (2017) Conserved roles of mouse DUX and human DUX4 in activating cleavage-stage genes and MERVL/HERVL retrotransposons. Nat. Genet. 49:925-934.

13. Lemmers RJ, et al. (2010) A unifying genetic model for facioscapulohumeral muscular dystrophy. Science 329:1650-1653.

14. Himeda CL, Jones TI, \& Jones PL (2015) Facioscapulohumeral muscular dystrophy as a model for epigenetic regulation and disease. Antioxid. Redox. Signal. 22:1463-1482.

15. Broucqsault N, et al. (2013) Dysregulation of 4q35- and muscle-specific genes in fetuses with a short D4Z4 array linked to facio-scapulo-humeral dystrophy. Hum. Mol. Genet. 22:4206-4214.

16. Ferreboeuf M, et al. (2014) DUX4 and DUX4 downstream target genes are expressed in fetal FSHD muscles. Hum. Mol. Genet. 23:171-181.

17. Jones TI, et al. (2012) Facioscapulohumeral muscular dystrophy family studies of DUX4 expression: evidence for disease modifiers and a quantitative model of pathogenesis. Hum. Mol. Genet. 21:4419-4430.

18. Rahimov F, et al. (2012) Transcriptional profiling in facioscapulohumeral muscular dystrophy to identify candidate biomarkers. Proc. Natl. Acad. Sci. 109:16234-16239. 
19. Rickard AM, Petek LM, \& Miller DG (2015) Endogenous DUX4 expression in FSHD myotubes is sufficient to cause cell death and disrupts RNA splicing and cell migration pathways. Hum. Mol. Genet. 24:5901-5914.

20. Tsumagari K, et al. (2011) Gene expression during normal and FSHD myogenesis. BMC Med. Genomics 4:67.

21. Bosnakovski D, et al. (2008) An isogenetic myoblast expression screen identifies DUX4mediated FSHD-associated molecular pathologies. EMBO J. 27:2766-2779.

22. Vanderplanck C, et al. (2011) The FSHD atrophic myotube phenotype is caused by DUX4 expression. PLoS One 6:e26820.

23. Homma S, Beermann ML, Boyce FM, \& Miller JB (2015) Expression of FSHD-related DUX4-FL alters proteostasis and induces TDP-43 aggregation. Ann. Clin. Transl. Neurol. 2:151-166.

24. Jiang S, et al. (2020) Single-nucleus RNA-seq identifies divergent populations of FSHD2 myotube nuclei. PLoS Genet. 16:e1008754.

25. van den Heuvel A, et al. (2019) Single-cell RNA sequencing in facioscapulohumeral muscular dystrophy disease etiology and development. Hum. Mol. Genet. 28:1064-1075.

26. Amini Chermahini G, Rashnonejad A, \& Harper SQ (2019) RNAscope in situ hybridization-based method for detecting DUX4 RNA expression in vitro. RNA 25:12111217.

27. Ferreboeuf M, et al. (2014) Nuclear protein spreading: implication for pathophysiology of neuromuscular diseases. Hum. Mol. Genet. 23:4125-4133. 
28. Tassin A, et al. (2013) DUX4 expression in FSHD muscle cells: how could such a rare protein cause a myopathy? J. Cell. Mol. Med. 17:76-89.

29. Zeng W, et al. (2016) Single-nucleus RNA-seq of differentiating human myoblasts reveals the extent of fate heterogeneity. Nuc. Acids Res. 44:e158.

30. Jagannathan S, et al. (2016) Model systems of DUX4 expression recapitulate the transcriptional profile of FSHD cells. Hum. Mol. Genet. 25:4419-4431.

31. Yao Z, et al. (2014) DUX4-induced gene expression is the major molecular signature in FSHD skeletal muscle. Hum. Mol. Genet. 23:5342-5352.

32. Shadle SC, et al. (2017) DUX4-induced dsRNA and MYC mRNA stabilization activate apoptotic pathways in human cell models of facioscapulohumeral dystrophy. PLoS Genet. 13:e1006658.

33. Katayama S, et al. (2018) Phylogenetic and mutational analyses of human LEUTX, a homeobox gene implicated in embryogenesis. Sci. Rep. 8:17421.

34. Jouhilahti EM, et al. (2016) The human PRD-like homeobox gene LEUTX has a central role in embryo genome activation. Development 143:3459-3469.

35. Shiomi K, et al. (2011) CDK4 and cyclin D1 allow human myogenic cells to recapture growth property without compromising differentiation potential. Gene Ther. 18:857-866.

36. Snider L, et al. (2009) RNA transcripts, miRNA-sized fragments and proteins produced from D4Z4 units: new candidates for the pathophysiology of facioscapulohumeral dystrophy. Hum. Mol. Genet. 18:2414-2430. 
37. Sikandar SS, et al. (2010) NOTCH signaling is required for formation and self-renewal of tumor-initiating cells and for repression of secretory cell differentiation in colon cancer. Cancer Res. 70:1469-1478.

38. Mainali KP, et al. (2017) Statistical analysis of co-occurrence patterns in microbial presence-absence datasets. PLoS One 12:e0187132. 


\section{Figure legends}

\section{Figure 1. Specific detection of DUX4 transcripts enriched in the FSHD myotube}

\section{nucleus}

A. Schematic diagram of mRNA transcripts for DUX4fl, the DUX4s isoform, DUX4 homologs (DUX4C and DUX1), and the positions and numbers of individual ZZ pairs for the previously published RNAScope $15 Z Z$ (26) and our $6 Z Z$ probe sets. The black regions represent $>99 \%$ homology to $D U X 4 f I$, which can be detected by corresponding ZZ pairs. The orange region in the 3' half of $D U X 4 C$ and DUX1 were confirmed not to crossreact with either $15 Z Z$ or $6 Z Z$. As at least $3 Z Z$ pairs are required for detectable signal, $15 Z Z$ is capable of efficient detection of $D U X 4 s$ and $D U X 4 c$. Our $6 Z Z$ probe set was designed to preferentially detect $D U X 4 f l$ and minimize the crossreactivity to $D U X 4 \mathrm{~s}$ and $D U X 4 c$. Numbers indicate nucleotide number from the 5 ' end of the transcripts. Nucleotide sequence comparison of four transcripts are shown in Supplemental Figure S1.

B. RNAScope costaining with $15 Z Z$ and $6 Z Z$ probe sets in FSHD myotubes. Colocalization of the nuclear foci detected by both $15 Z Z$ and $6 Z Z$. The lower panel is a magnification of the boxed region in the top panel. White arrowheads indicate the colocalization of both probe sets in the cytoplasm. Scale bar $=10 \mu \mathrm{m}$.

C. Additional nuclear foci in control and FSHD myotubes.

Weak but distinct nuclear foci were observed in control cells with 15ZZ. No signal was observed with $6 Z Z$ in control cells. Scale bar $=10 \mu \mathrm{m}$.

D. Immunofluorescent staining of DUX4 protein (red) and RNAScope detection of DUX4 
transcript using $6 Z Z$ probes (green) in primary FSHD myotubes at day 7 of differentiation. DAPI is in blue. White dashed lines indicate the boundary of a myotube with positive DUX4 antibody staining signal. Scale bar $=10 \mu \mathrm{m}$.

\section{Figure 2. DUX4 transcripts upregulated in FSHD myotubes}

A. Bright field images of immortalized clonal FSHD2 myoblast differentiation on day 0 and day 4. Scale bar=100 $\mu \mathrm{m}$

B. RNAScope costaining of DUX4 $15 Z Z$ (red) and $6 Z Z$ (green) probes in immortalized FSHD myotubes. White arrow indicates colocalization of probes. The lower panel is a magnification of the top panel. $15 Z Z$ staining shows more foci and brighter signal similar to RNAScope staining in patient primary cells. Scale bar $=20 \mu \mathrm{m}$.

C. Characterization of DUX4 6ZZ RNAScope in day 3 immortalized control and FSHD myotubes. DUX4 staining usually appears in the nucleus and has around 1-2 bright foci. Around $2.8 \%$ of 414 FSHD2 myotubes contained a positive DUX4 RNAScope signal. Scale bar $=20 \mu \mathrm{m}$.

D. Detection of DUX4 protein by immunofluorescent staining and DUX4 RNA by RNAScope. DUX4 RNAScope (green) is combined with immunofluorescence using antibody against DUX4 (red) in immortalized FSHD myotubes at day 3 of differentiation. DAPI is in blue. White dashed line indicates the boundary of a myotube with positive DUX4 antibody staining signal. Arrow indicates nuclei with DUX4 transcripts. Scale bar $=10 \mu \mathrm{m}$.

Figure 3. Comparison with DUX4 target gene expression 
A. RNAScope staining of DUX4 target genes LEUTX, KDM4E, SLC34A2, and ZSCAN4. Unlike DUX4, staining of target genes is spread along the myotube and in the cytoplasm. Approximately $2 \%$ of myotubes showed positive RNAScope target gene signal. Scale bar $=20 \mu \mathrm{m}$.

B. Costaining of LEUTX RNAScope (green) and LEUTX protein immunofluorescence staining (red) in control and FSHD myotubes on differentiation day 6. The middle panel shows a typical example of RNA and protein colocalization while the bottom panel shows an example of weak protein staining without RNA. Numbers of myotubes with corresponding patterns in the total myotubes counted are shown in parentheses. Percentages of myotubes with LEUTX RNA only, LEUTX RNA and protein, or LEUTX protein only are shown. Scale bar $=20 \mu \mathrm{m}$.

Figure 4. Dynamic relationship between DUX4 and target gene expression during myotube differentiation

A. Bright field images of immortalized FSHD myoblast differentiation into myotubes on days 3-13. Around day 5, we find greater myotube detachment. Scale bar $=100 \mu \mathrm{m}$ B. Quantification of time course RNAScope triple staining of DUX4, LEUTX, and $K D M 4 E$ transcripts. Error bars indicate the standard deviation from the mean of three independent experiments. The actual number counts are shown in Supplemental Figure S3.

C. Frequencies of myotubes expressing DUX4 only, DUX4 and target genes, or target genes only change during differentiation. Replotting the data in (B) for the number of myotubes containing DUX4 transcripts only, transcripts of DUX4 plus target gene(s), or 
transcripts of target gene(s) only as indicated. Y-axis is the total number of positive myotubes counted.

D. Frequency of LEUTX expression in DUX4-expressing myotubes decreases later in differentiation. The ratios between the percentage of LEUTX-expressing (LEUTX $(+))$ myotubes in the entire DUX4-expressing (DUX4(+)) myotubes and the percentage of LEUTX(+) myotubes in myotubes with no DUX4 expression $(D U X 4(-))$ at different days after differentiation were calculated based on the RNAScope data in (B). At day3, the frequency of DUX4(+) myotubes to co-express LEUTX is $\sim 20-60$ fold higher than the frequency of $D U X 4(-)$ myotubes to express LEUTX. Later in differentiation (days 7 and 9), the ratios drop significantly, indicating that the frequency of LEUTX expression without $D U X 4$ expression becomes more significant. The error bars represent the standard deviation of the mean of 3 independent experiments. The significance was evaluated by student t-test. The $p$ values were calculated comparing to day 3. $p<0.05$ was considered significant.

Fig. 5. KDM4E expression is regulated by DUX4 target transcription factors A. Individual timecourse RNAScope analysis of DUX4, LEUTX, and KDM4E in FSHD myotubes for the indicated days of differentiation from Figure 4B. These include all the positive myotubes for each transcript.

B. Comparison of correlation between KDM4E and DUX4/LEUTX expression based on the RNAScope data in Figure 4B. The high $\varphi$ values indicate strong positive relationship between KDM4E and LEUTX RNA expression for the entire duration of the timecourse, with a perfect positive correlation $(\varphi=1)$ on day 4 . After that, the $\varphi$ values decrease but 
are more significant than those between KDM4E and DUX4 expression until day 13 . Pvalues (unpaired, two-tailed t test) are for the correlation differences of indicated genes at the same day.

C. The effect of DUXA or LEUTX depletion on KDM4E expression in FSHD myotubes. . KDM4E gene expression in control, DUXA, or LEUTX shRNA-treated myotubes on day 4 and day 6 was measured by RT-qPCR. The signal was normalized to GAPDH. The bar graph shows the average fold change of three independent experiments for each knockdown compared to the control shRNA-treated cells on day 4 or day 6 as indicated. Student t-test was used to calculate p-values. ${ }^{*} p$-value $<0.05,{ }^{* *} p$-value $<0.01$.

D. Analysis of depletion efficiency of DUXA and LEUTX shRNA by RT-qPCR. Student ttest was used to calculate p-values for expression of DUXA or LEUTX in corresponding shRNA-treated myotubes compared to shRNA control on days 4 and 6 of differentiation. ${ }^{*} p$-value $<0.05,{ }^{* * *} p$-value $<0.001,{ }^{* * * *} p$-value $<0.0001$

E. A schematic diagram depicting the proposed relationship between DUX4 and the target genes. DUX4 expression is important for induction of DUXA, LEUTX and KDM4E during early FSHD myoblast differentiation. Once expressed, LEUTX contributes to KDM4E upregulation. DUXA activates LEUTX and KDM4E (directly and/or possibly through LEUTX). 
Figure 1
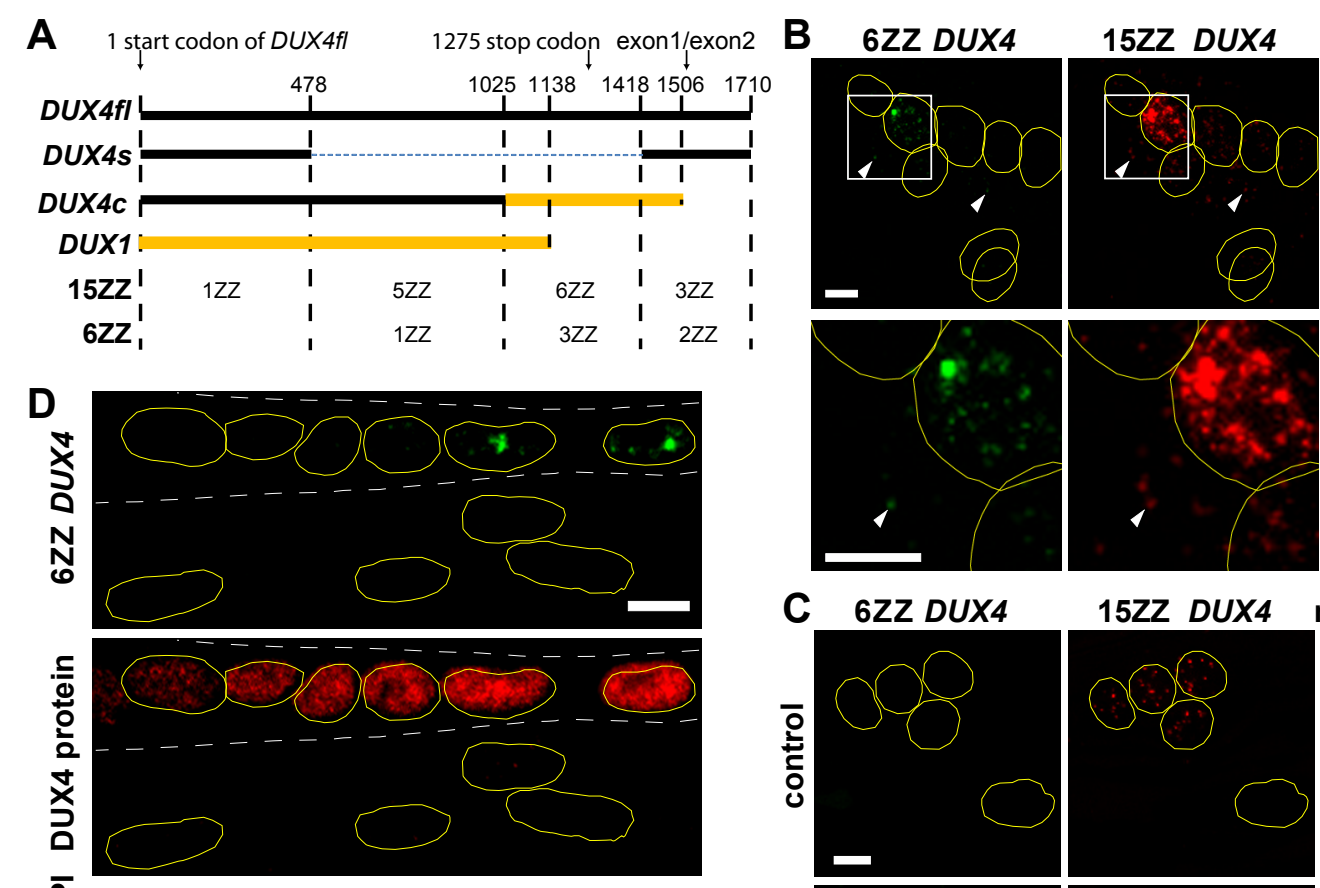

merged with DAPI
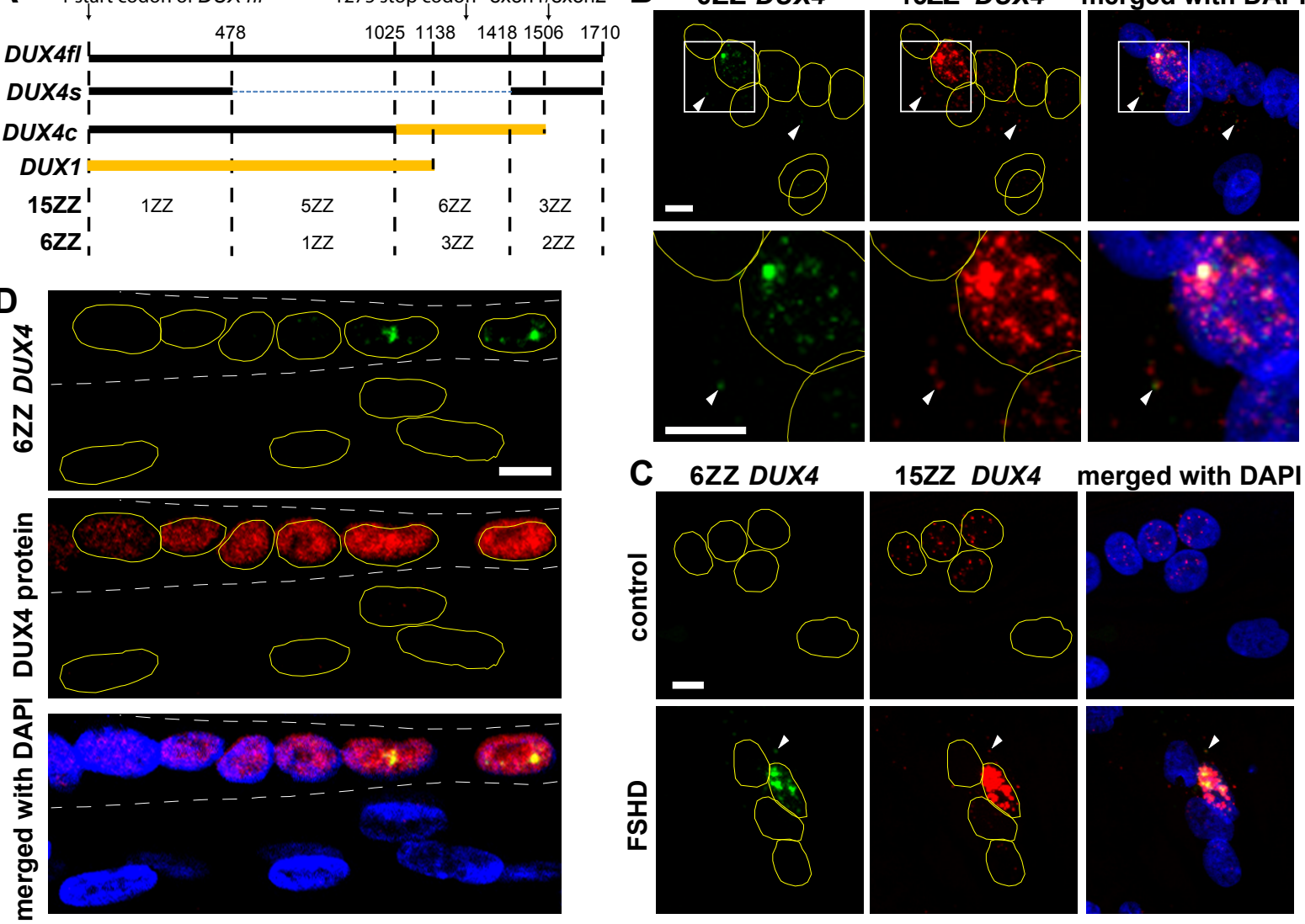
Figure 2

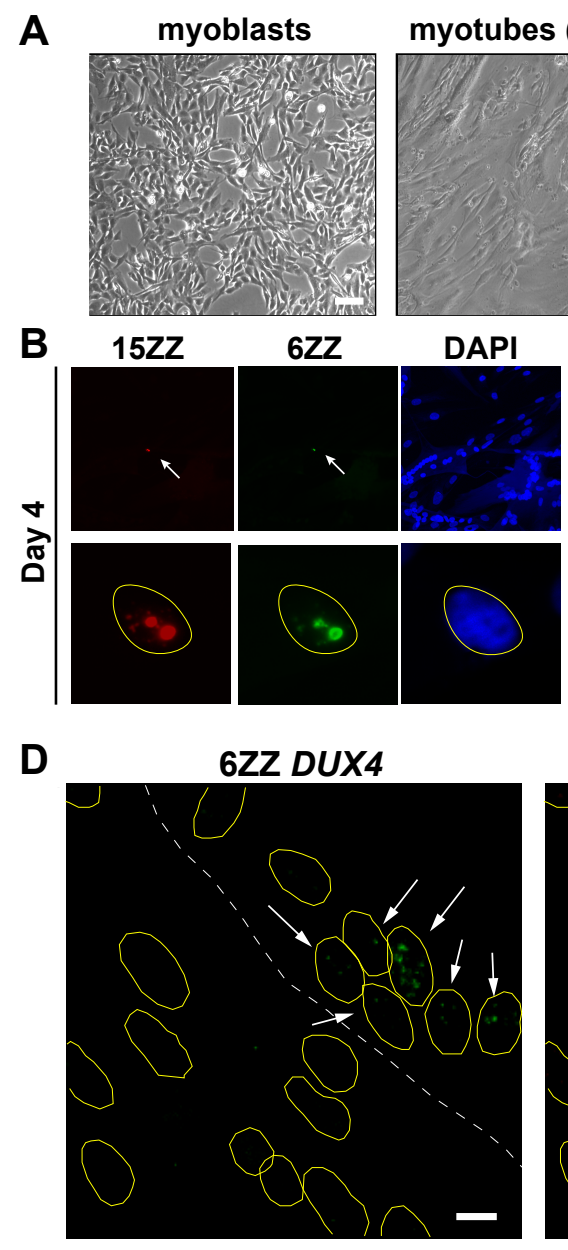

C
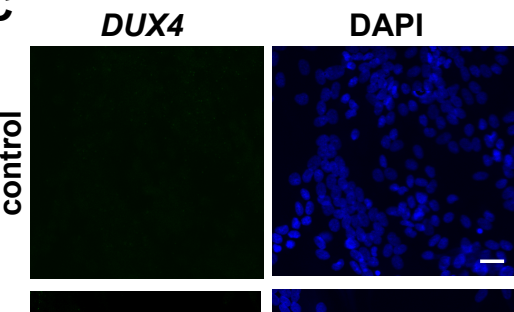

merged

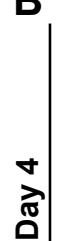
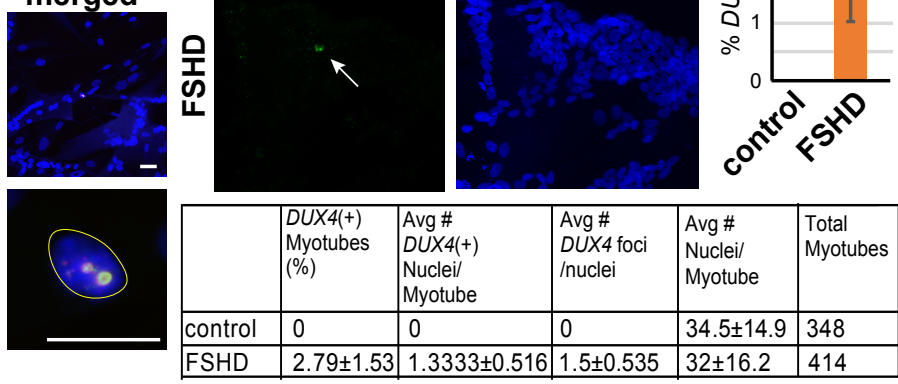

D

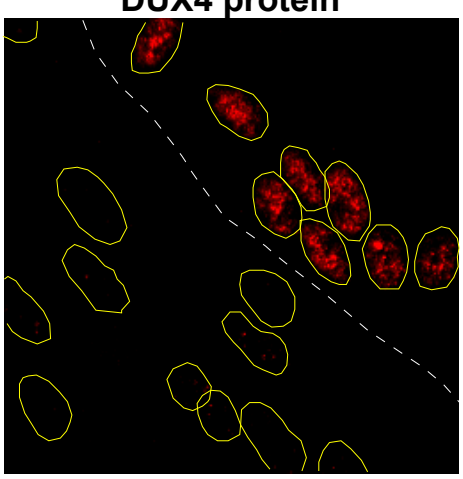

merged with DAPI

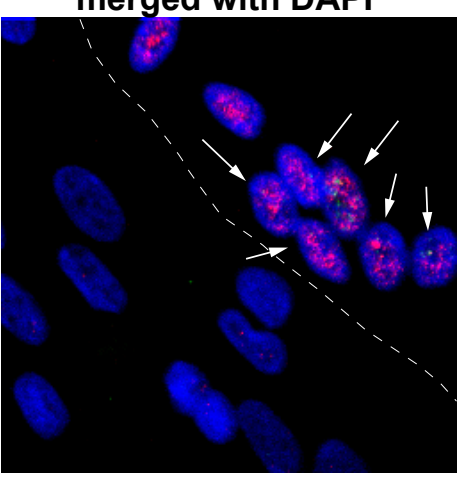


Figure 3

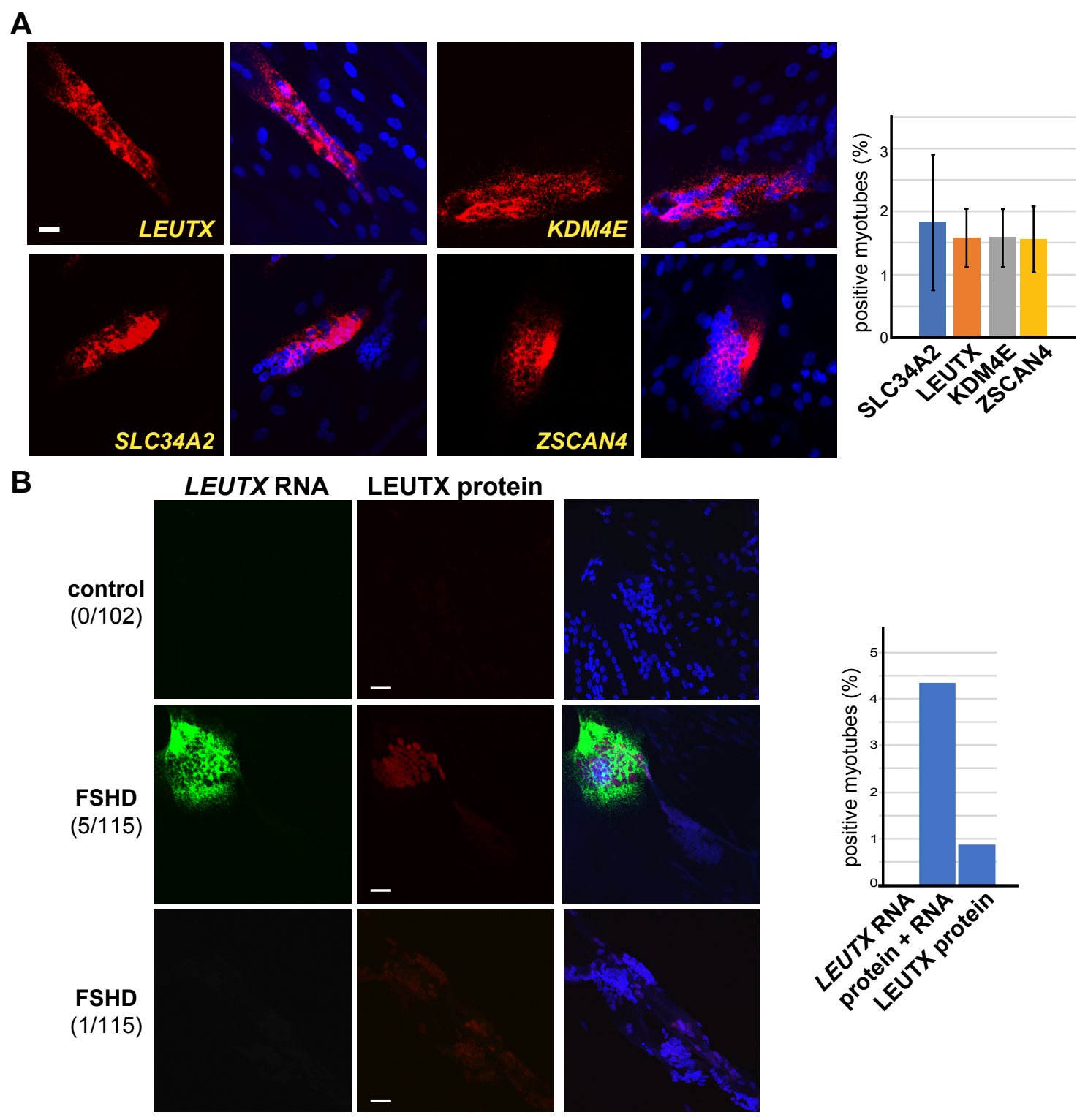


Figure 4
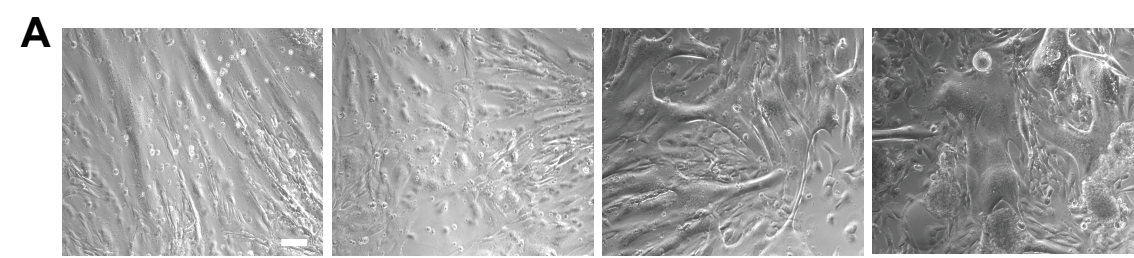

D5

D7

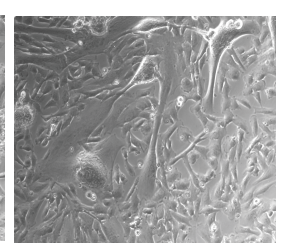

D9

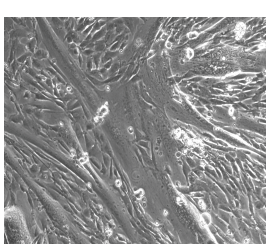

D13

B

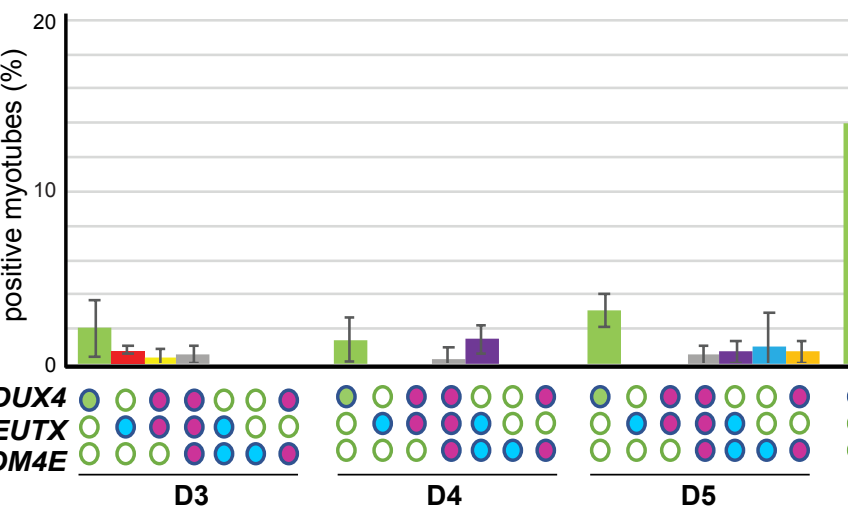

C

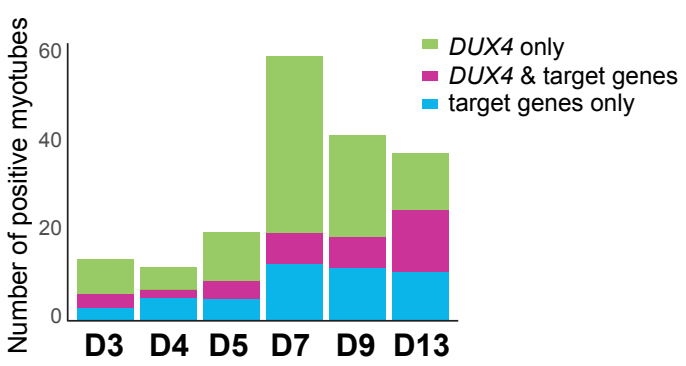

D

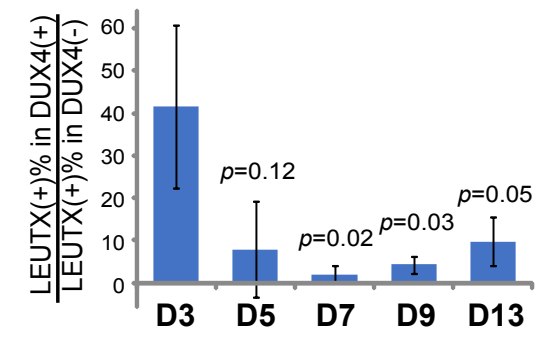


Figure 5

A

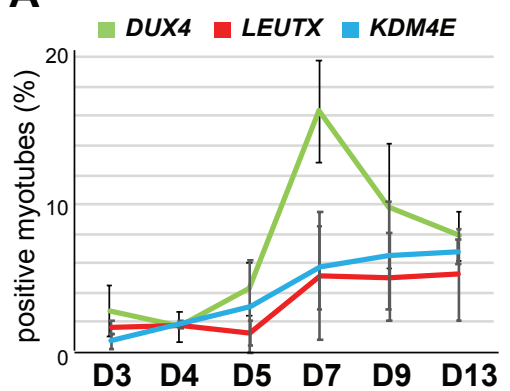

C

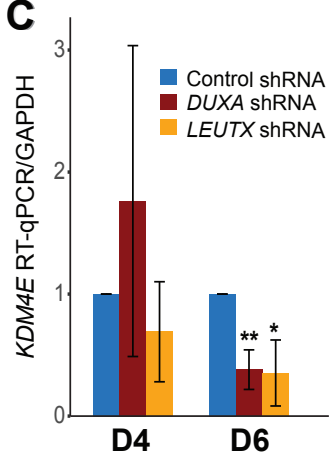

D
B
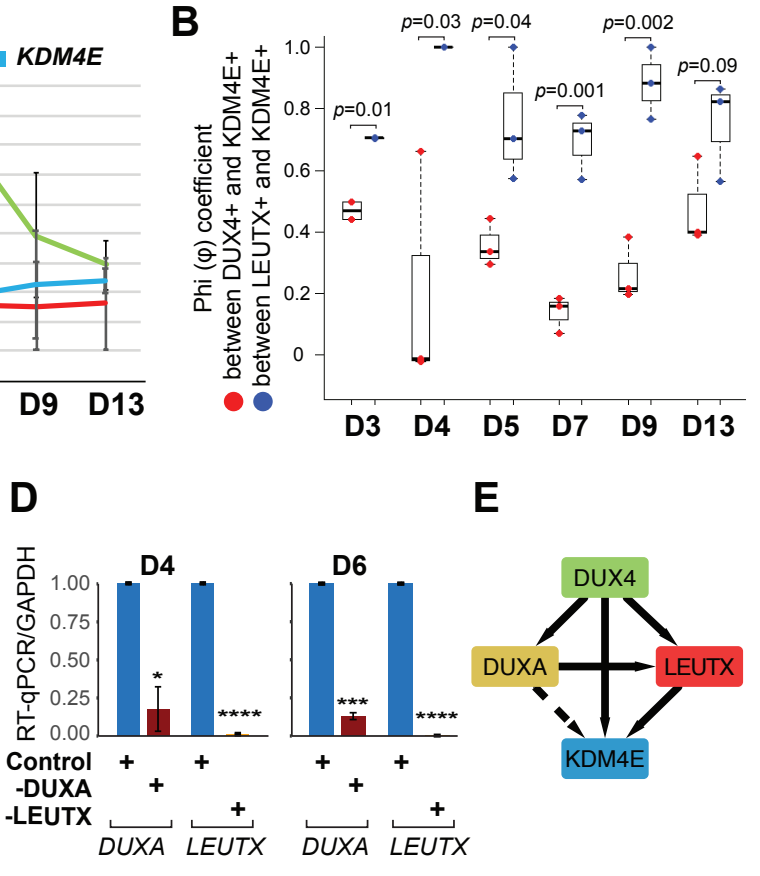

E

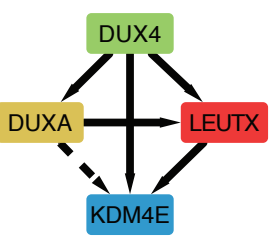




\section{Supplemental Table S1. PCR primers}

KDM4E_F
KDM4E_R
LEUTX_F
LEUTX_R
DUXA_F
DUXA_R
GAPDH_F
GAPDH_R
5'-TCCCCAGAACACGAGTCATAC-3'

5'-TTGGGTGGAATTACCTTGGCA-3'

5'-GGGAAACTGGCTTCAAAGCTA-3'

5'-TGATGGCCGTGTCTGCATTT-3'

5'-GCCTTACCCAGGTTATGCTACC-3'

5'-TGGAATCCGTGCCTAGCTCTT-3'

5'-TCGACAGTCAGCCGCATCT-3'

5'-CTAGCCTCCCGGGTTTCTCT-3' 


\section{Supplemental Figure S1}

Start codon of DUX4fI ORF

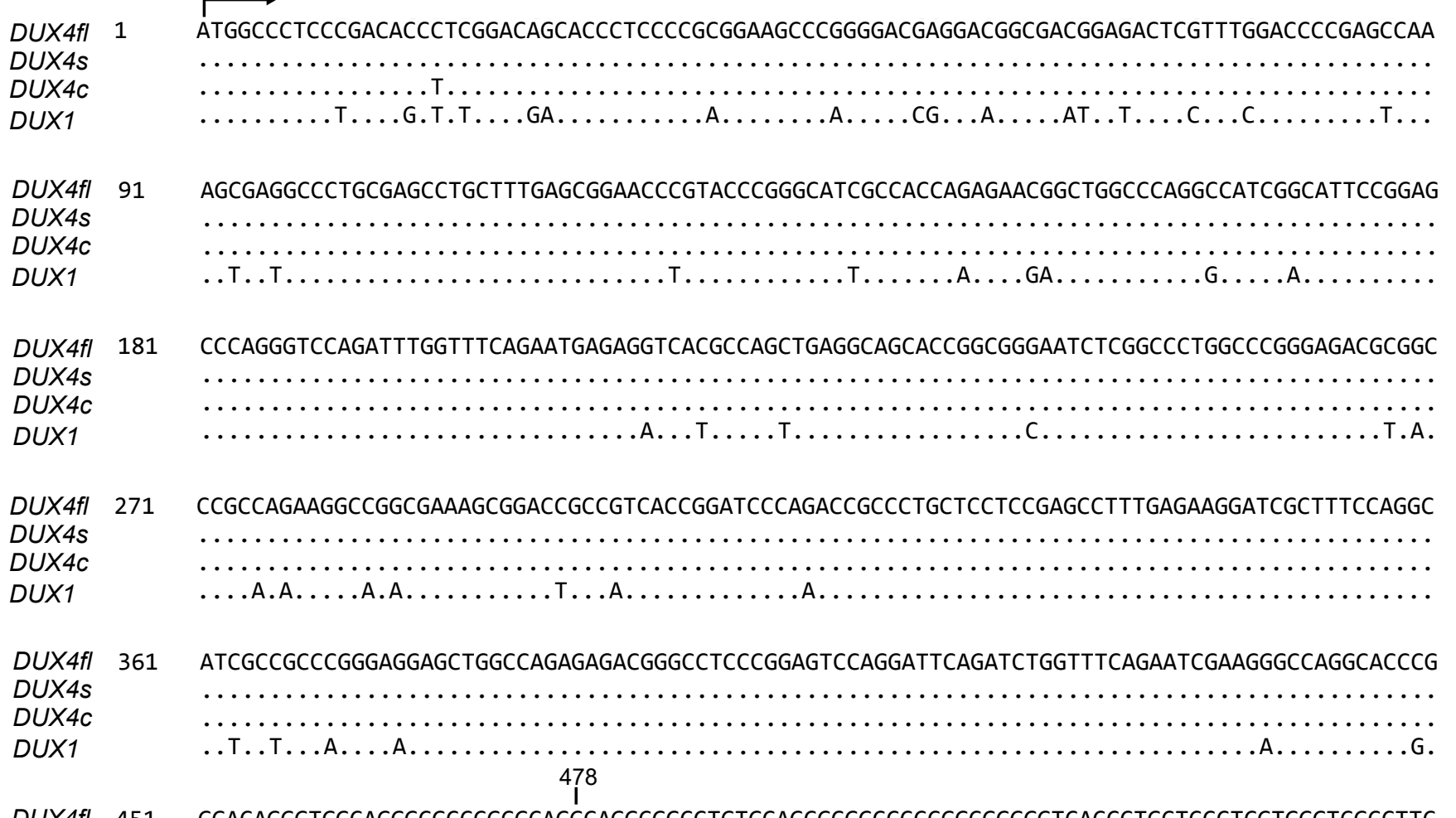

DUX4fl 451 GGACAGGGTGGCAGGGCGCCCGCGCAGGCAGGCGGCCTGTGCAGCGCGGCCCCCGGCGGGGGTCACCCTGCTCCCTCGTGGGTCGCCTTC DUX4s DUX4C DUX1

DUX4fI DUX4s DUX4c DUX1

DUX4fI DUX4s DUX4C $D U X 1$ $\ldots \ldots \ldots \ldots \ldots \ldots \ldots \ldots \ldots \ldots \ldots \ldots$

GCCCACACCGGCGCGTGGGGAACGGGGCTTCCCGCACCCCACGTGCCCTGCGCGCCTGGGGCTCTCCCACAGGGGGCTTTCGTGAGCCAG

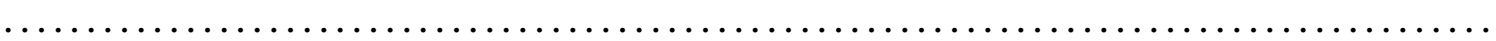

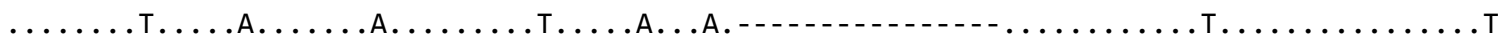

631 GCAGCGAGGGCCGCCCCCGCGCTGCAGCCCAGCCAGGCCGCGCCGGCAGAGGGGATCTCCCAACCTGCCCCGGCGCGCGGGGATTTCGCC

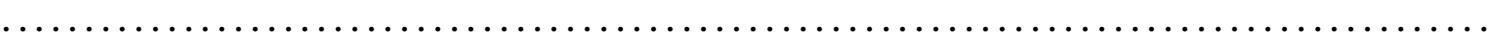
.G.TT......T...T.T...C.T.

.A.......

A. A.AT. TACGCGCCCGGCTCCTCCGGACGGGCGCTCTCCACCCTCAGGCTCCTCGCTGGCTCCGCACCCGGCAAAAGCGGGAGACCGG

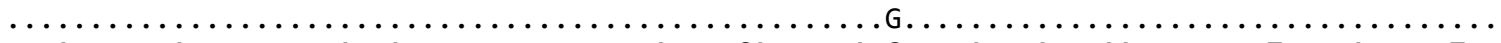
DUX1

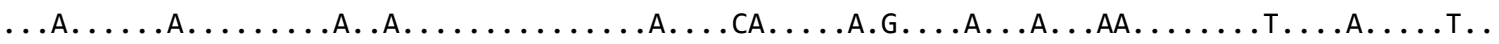




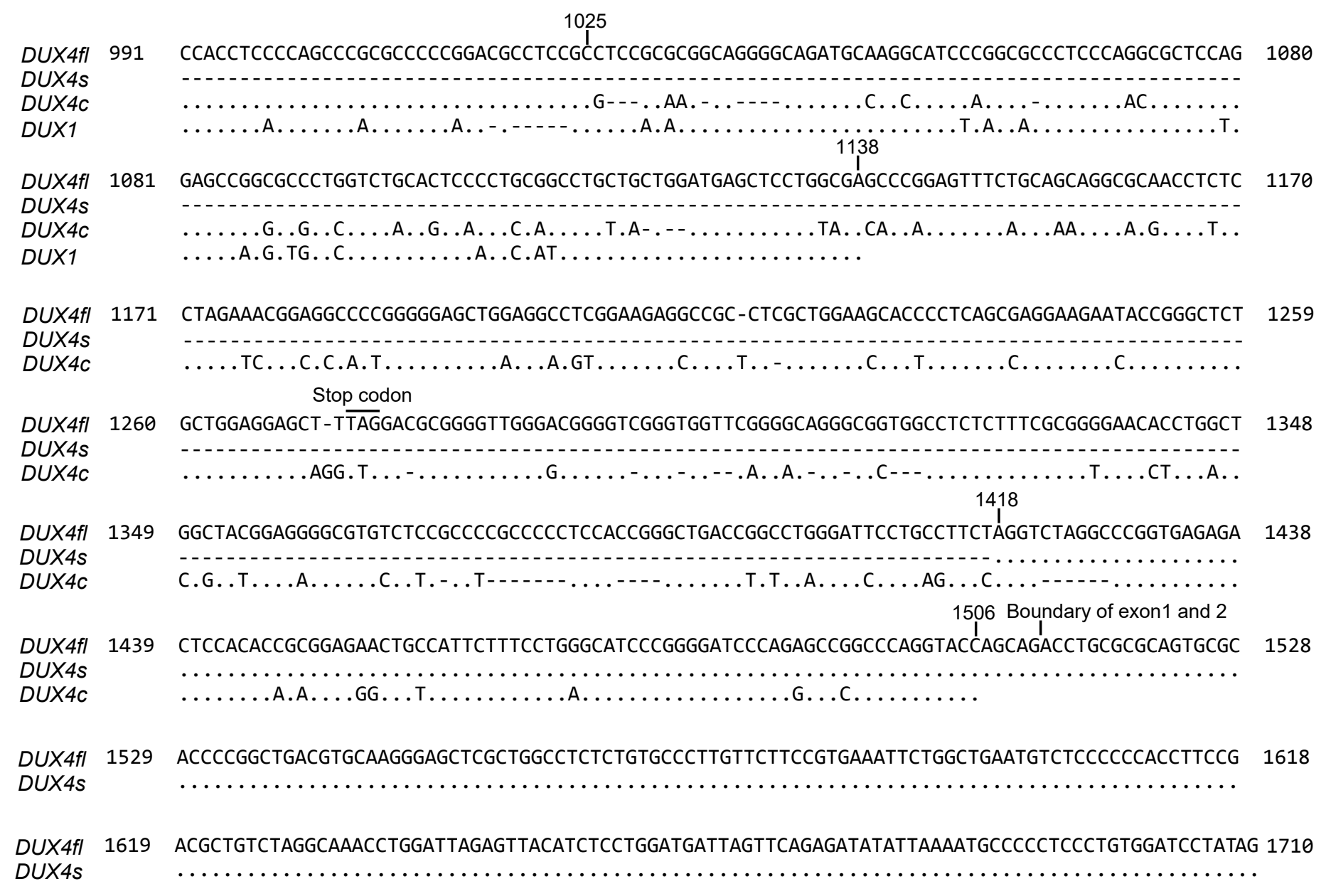

Supplemental Figure S1. Sequence comparison of $D U X 4 f l, D U X 4 s, D U X 4 c$ and $D U X 1$ transcripts. $D U X 4 s, D U X 4 c$ and $D U X 1$ transcripts alignments are shown in relation to $D U X 4 f l$. Identities are displayed as dots (.), with mismatches displayed as single letter abbreviations. Dash marks (-) indicate deleted regions. The start codon, stop codon and exon 1/exon 2 boundary of $D U X 4 f l$, as well as the regions boundaries in Figure $1 \mathrm{~A}$ were labeled on top of $D U X 4 f /$ sequence. Nucleotide numbering was based on DUX4fI transcript with accession number NM_001306068.2 in the NCBI database. 


\section{Supplemental Figure $\mathbf{S 2}$}

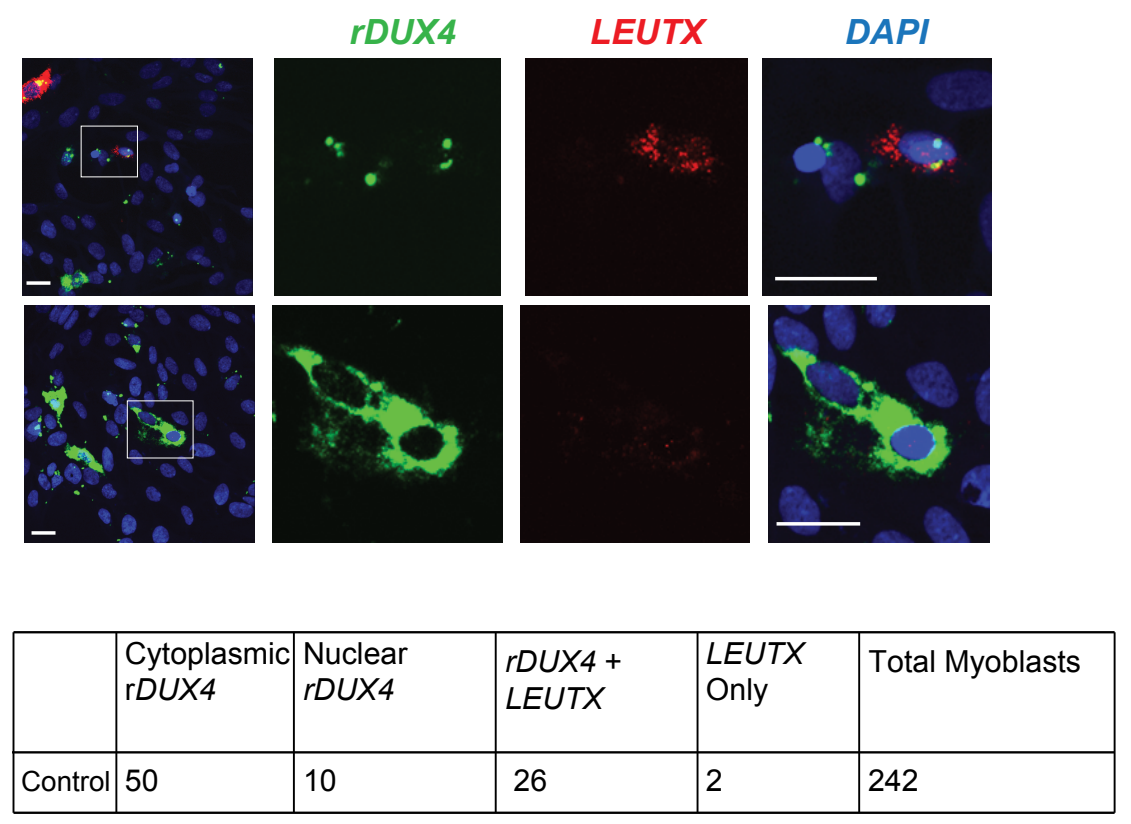

Supplemental Figure S2. RNAScope analysis of the transiently transfected recombinant DUX4 (rDUX4) and the endogenous LEUTX RNA expression in immortalized control myoblasts. Example images of different $r D U X 4$ RNA localization patterns are shown. The boxes in the first column indicate where the image is zoomed in. Table underneath shows the quantification of the number of myoblasts with different patterns of $r D U X 4$ and LEUTX RNA signals. Majority of $r D U X 4$-transfected myoblasts exhibited strong cytoplasmic rDUX4 RNA signals. LEUTX expression is not always seen in $r D U X 4$-expressing cells. Scale bar $=20 \mu \mathrm{m}$. 


\section{Supplemental Figure \$3}

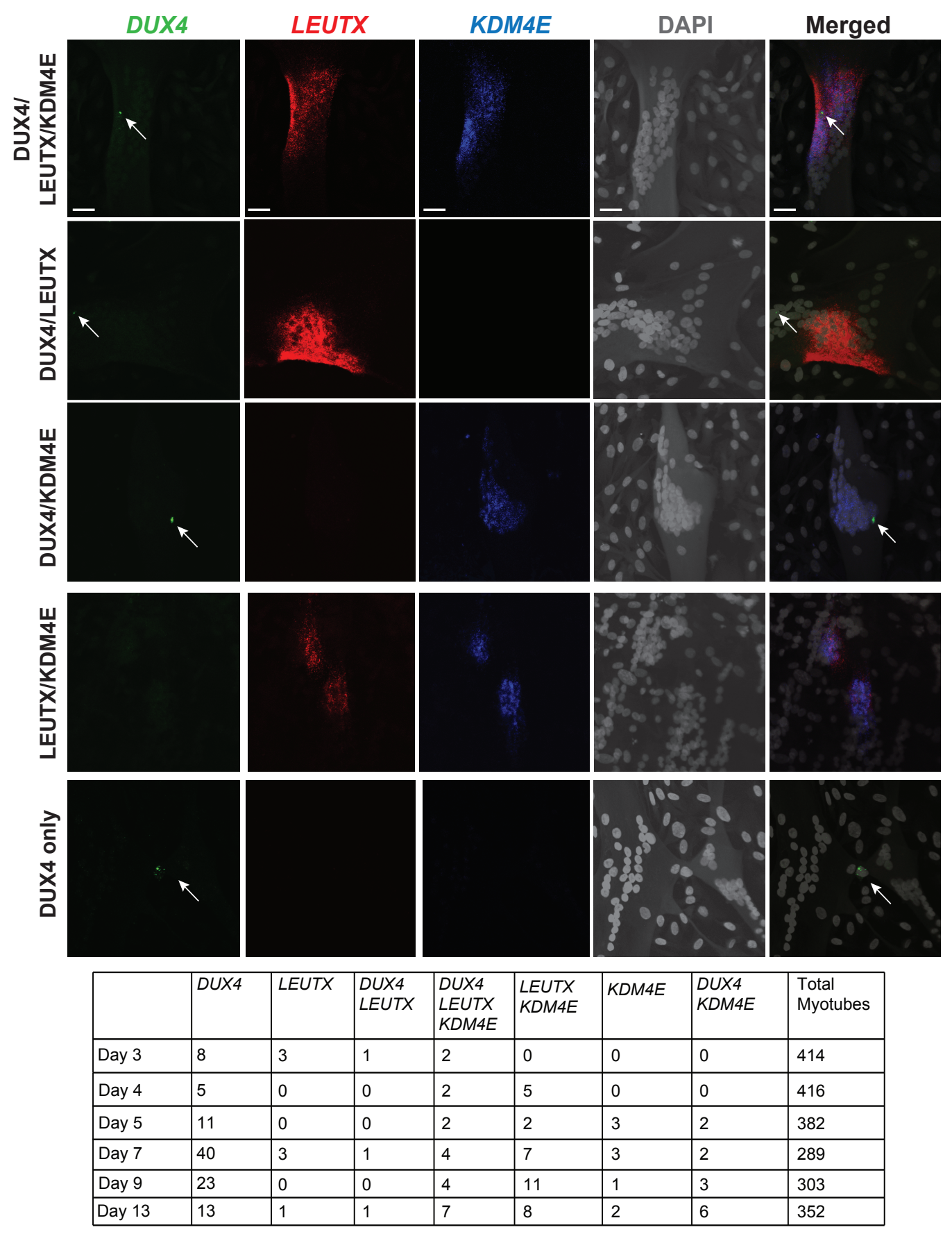

Supplemental Figure S3. Representative images of myotubes triple stained with RNAScope for DUX4, LEUTX, and KDM4E RNA during differentiation providing data for Figure 4. White arrows indicate DUX4 transcript-positive nuclei. The table shows the total counts from the three replicates. Scale bar $=20 \mu \mathrm{m}$. 


\section{Supplemental Figure S4}

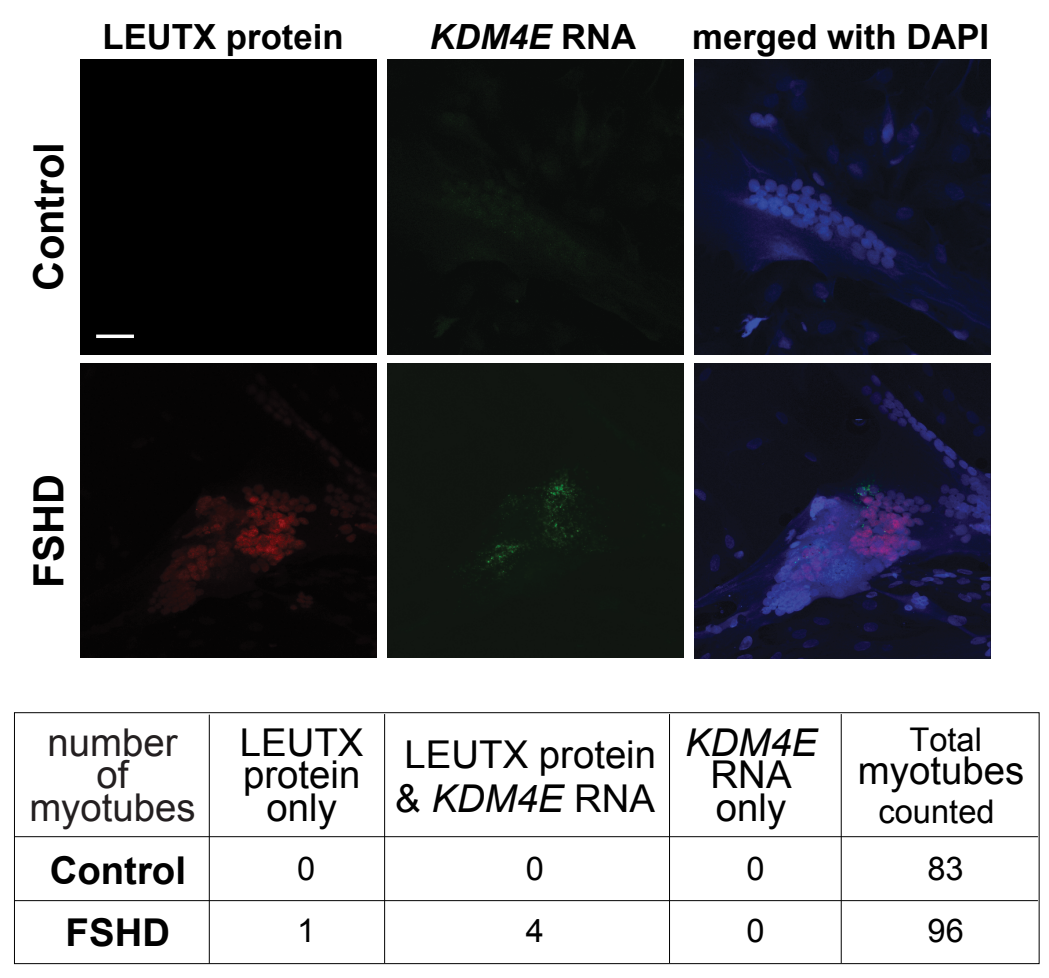

Supplemental Figure S4. Immunofluorescence staining of LEUTX protein and KDM4E RNAScope on differentiation day 6 in FSHD immortalized myotubes. All observed KDM4E transcripts colocalized with LEUTX protein. Scale bar $=20 \mu \mathrm{m}$. 


\section{Supplemental Figure S5}

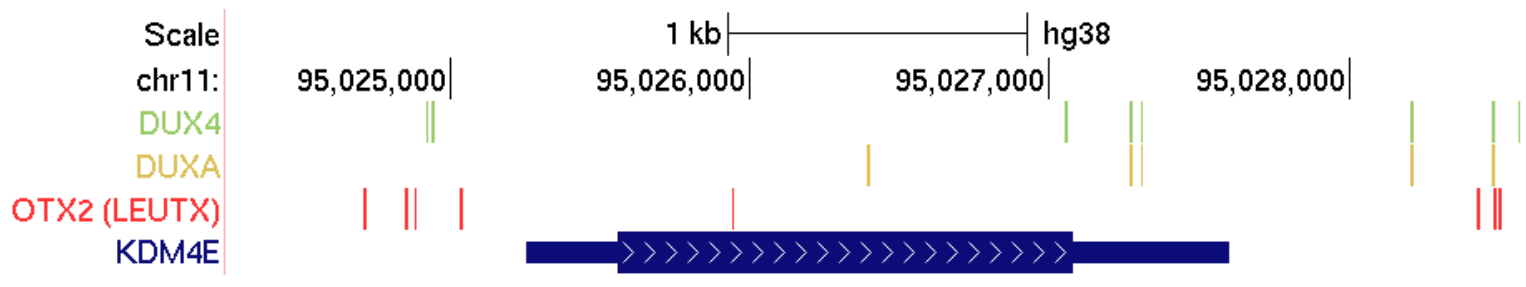

Supplemental Figure S5. TF binding motifs at the promoter of KDM4E. Binding motifs for DUX4, DUXA and the putative LEUTX motif (OTX2) within $1 \mathrm{~kb}$ upstream and $0.5 \mathrm{~kb}$ downstream of the transcription start site (TSS) for KDM4E. We used binding motifs from HOCOMOCO v11 (1) for DUX4, DUXA and OTX2 as input into HOMER (version 4.10) using the scanMotifGenomeWide.pl command for hg38 (2). Since no binding motif was available for LEUTX, we used the motif for OTX2, another PRD-like homeobox TF with a similar homeodomain, which was experimentally validated to function as LEUTX binding site (3). Visualization was done on the UCSC genome browser (4) using GENCODE v28 for the KDM4E gene model.

1. Kulakovskiy IV, et al. (2018) HOCOMOCO: towards a complete collection of transcription factor binding models for human and mouse via large-scale ChIP-Seq analysis. Nucleic Acids Res. 46:D252-D259.

2. Heinz S, et al. (2010) Simple combinations of lineage-determining transcription factors prime cis-regulatory elements required for macrophage and B cell identities. Mol. Cell 38:576-589.

3. Katayama S, et al. (2018) Phylogenetic and mutational analyses of human LEUTX, a homeobox gene implicated in embryogenesis. Sci. Rep. 8:17421.

4. Kent WJ, et al. (2002) The human genome browser at UCSC. Genome Res. 12:996-1006. 